\title{
Ai Enabled Blind Spot Detection Using Rcnn Based Image Processing
}

\author{
Elizabeth Angela M, Thilagavathy K, Soundharya R, M Somasundaram
}

\begin{abstract}
Due to the rapid increase in the rate of road accidents and traffic density, modern automobiles are equipped with intelligent systems like Adaptive cruise control and Lane Departure Warning System. Therear-view mirror can be effective to observe a limited range only and there are zones that cannot be viewed. This region is referred to as the blind spot. Therefore, we present a method to detect the vehicles from the side and the rear for Blind Spot Detection with vision system incorporating RCNN. Blind spot detection is a key technology among driver aids that provides 360 degrees of electronic coverage around the car during motion.The methodology presented in this paper uses two stereo cameras as input devices which constantly capture the images at the blind spot area and the information is passed to the main controlling unit. Potholes are also detected and the alert is sent to the nearby vehicle. The incorporation of Artificial Intelligence would help in enhancing the picture quality and blur or cancel the background images probable of misreading the target image. RCNN is used for the vehicle detection and for evaluating the relative distance between the vehicles.This technology allows us to provide a realistic environment for commercial vehicle drivers as they can't monitor the side and rear-view mirrors all the time, making the whole driving experience more comfortable.
\end{abstract}

Keywords-Blind spot, RCNN, Potholes, Artificial Intelligence

\section{INTRODUCTION}

ADAS

Advanced driver-assistance systems (ADAS) are systems designed to assist the user in the driving process. It provides an intelligent human-machine interface, thereby increasing car safety. This system will contribute in reducing human errors. ADAS technology can help in detecting and classifying objects, alerting the driver of risky road conditions and, in some cases, slow down or stop the vehicle. ADAS is useful for applications like blind spot monitoring, lane-keep assistance and forward collision warning.

ADAS systems range on the spectrum of active or passive. A passive system alerts the driver of a potentially dangerous situation such that he corrects it. For example, Lane Departure Warning (LDW) alerts the driver of unintended lane departure. Forward Collision Warning (FCW) detects the collision underexisting circumstance and alerts the driver to apply brakes in order to avoid the collision. On the other hand, active safety systems take

Revised Manuscript Received on July 22, 2019.

Elizabeth Angela M, Department of Electronics and Communication Engineering,R.M.K Engineering College,Chennai-601 206.

Thilagavathy K, Department of Electronics and Communication Engineering,R.M.K Engineering College,Chennai-601 206.

Soundharya R, Department of Electronics and Communication Engineering,R.M.K Engineering College,Chennai-601 206.

M Somasundaram Department of Electronics and Communication Engineering,R.M.K Engineering College,Chennai-601 206. action automatically. Automatic Emergency Braking (AEB) detects collision and brakes without any driver intervention.

\section{BLIND SPOT DETECTION}

A blindspot in a vehicle can be defined as an area around the vehicle that cannot be directly observed by the driver while under control. Blind spot detection system consists of two cameras mounted on either aspect of side-view mirrors which is kept at an angle that the side mirrors cannot cover. If the driver under control doesn't notice the presence of these vehicles, it may lead to abrupt lane changing resulting in accidents. Therefore, we employ Region based Convolutional Neural Network (RCNN), a deep learning algorithm to detect and classify the vehicles by comparing with the image database.

\section{POTHOLE DETECTION}

Potholes are hollows in a road surface caused by wear or subsidence. These potholes are detected and the information is conveyed to the slave device via UART serial communication using ZigBee module.

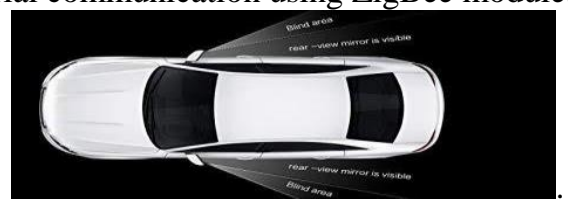

Fig 1 BLIND SPOT IN VEHICLES

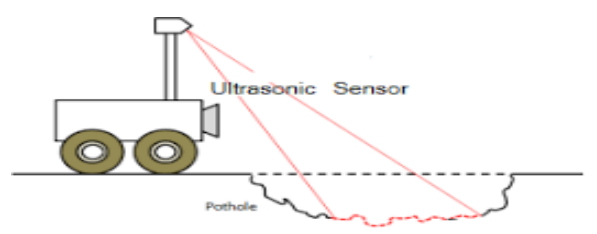

Fig 2 : POTHOLE DETECTION

\section{EXISTING METHOD}

A typical blind spot observance system needs an electronic detection device mounted on the perimeters of the automotive (often within the neighborhood of the external rear read mirrors or close to the rear bumpers) that generates electronic magnetism waves. Optical sensors analyze the particular flow of traffic to spot any

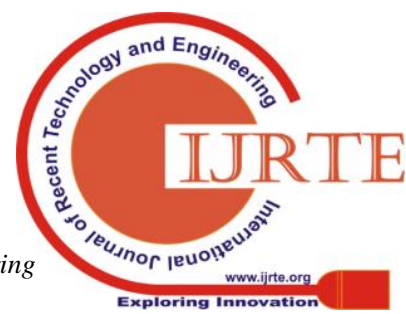




\section{Ai Enabled Blind Spot Detection Using Renn Based Image Processing}

objects within the blind spot of the vehicle.

Software based Blind spot detection systems include multiple target capability, open road testing, video integration, BSD warning, free analysis software and multiple contact points.

A special convexo-concave mirror can be placed at the corner of the available car mirror. But the disadvantage of this method is that the driver has to keep looking at the mirrors and have frequent distractions from the road.

Through this process we are able to stop the accident before it occurs. The ultrasonic sensor is used to detect the pothole and indicate it to the user on LCD and the information is shared to the nearby vehicles.

\section{PROPOSED SYSTEM}

In the proposed system, cameras are placed on both sides of the mirror to capture images of the vehicles which are not under the range of side view mirrors. This is transferred to

\section{RCNN ALGORITHM}

\section{Fig 3: RCNN}

MATLAB for image processing. In MATLAB, the captured image will be pre-processed, and it will identify the vehicle and its position using RCNN. All the processing done by the MATLAB will be updated on the microcontroller (Arduino). This will ensure the user to identify the side from which the vehicle is approaching. The real time video feed of the blind spot area with the necessary information about the vehicle is displayed on an LCD display.

Another aspect is to check for potholes and an alert message is given to the nearby vehicle through the ZigBee module.

Instead of analyzing a large number of regions, the RCNN algorithm proposes a bunch of boxes in the image and checks if any of these boxes contain the objects to be detected via selective search.

The regions that form an object are varying scales, colors, textures, and enclosure. Selective search identifies the patterns in the image and proposes various regions.

It will then combine the similar regions to form a larger region. Finally, these regions then produce the final object locations consisting of the Region of Interest.

As this is slow, we employ fast RCNN which uses a single model to extract the features from the different regions, divides them into appropriate classes, and returns the boundary boxes for the identified classes simultaneously.

Fast RCNN replaces the selective search method with region proposal network which results in the much faster

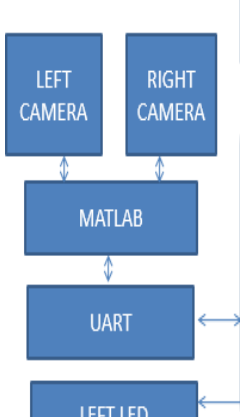
POWER SUPPLY
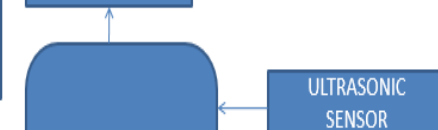

algorithm. The input image resolutions are high when compared to other algorithms. The time cost of generating region proposals or prediction is very low. Data augmentation is an added advantage to enhance the training of convolutional neural networks.

\section{CONCLUSION}

This paper has given an idea for vehicle detection and classification at the blind spot region using a deep learning algorithm (RCNN). It also provides the distance details between the host vehicle and object detected such that the user can take appropriate decisions such as lane departure warning, rear and front parking sonar, the rear traffic alert and parking. Blind spot detection depends on the angle at which we set the camera at the side mirror. The angle should be such that it covers the area not covered by the side mirrors. We've taken an implemented when a video is given as input and when a real time object is placed.It is one of ........................ mitigate possible road accidents in the near future which may result in loss of life and property.

\section{REFERENCES}

1. Amir Vahid Dastjerdi and Rajkumar Buyya, "Fog Computing: Helping the Internet of Things Realize Its Potential", IEEE Communications Society, August 2016.

2. Aref Meddeb, "Internet of Things Standards: Who Stands Out from the Crowd?", IEEE Communications Magazine - Communications Standards Supplement, July 2016.

3. Constantinos Kolias and Angelos Stavrou, Irena Bojanova, and Richard Kuhn, "Learning Internet of Things Security Hands-on", Copublished by the IEEE Computer and Reliability Societies, January/February 2016.

4. Dusit Niyato, Dinh Thai Hoang, Nguyen Cong Luong, Ping Wang, Dong In Kim, and Zhu Han, "Smart Data Pricing Models for the Internet of Things: A Bundling Strategy Approach", IEEE Network, March/April 2016

5. David Park, "The Quest for the Quality of Things: Can the Internet of Things deliver a promise of the quality of things?", IEEE Consumer Electronics Magazine, April 2016

6. Daqiang Zhang, Laurence Tianruo Yang, Min Chen, Shengjie Zhao, Minyi Guo, and Yin Zhang, "Real-Time Locating Systems Using the Active RFID for the Internet of Things", IEEE Systems Journal, Vol. 10, No. 3, September 2016.

7. David Metcalf, Sharlin T. J. Milliard, Melinda Gomez, and Michael Schwartz, "Wearables and the Internet of Things for Health", IEEE Pulse, September / October 2016.

8. Glenn Parsons, "The Internet of Things", IEEE Communications Magazine, July 2016.

9. Guiou Kobayashi, Maria Eunice Quilici-Gonzalez, Mariana Claudia Broens, and José Artur Quilici-Gonzalez, "The Ethical Impact of the Internet of Things in Social Relationships", IEEE Consumer Electronics Magazine, July 2016.

10. Huadong Ma, Liang Liu, Anfu Zhou, and Dong Zhao, "On the Networking of Internet of Things: Explorations and Challenges", IEEE Internet of Things Journal, Vol. 3, No. 4, August 2016.

11. Huadong Ma, Liang Liu, Anfu Zhou, and Dong Zhao, "On the Networking of Internet of Things: Explorations and Challenges", IEEE Internet of Things Journal, Vol. 3, No. 4, August 2016.

12. Jonathan Margulies, "Garage Door Openers: An Internet of Things Case Study", IEEE Computer and Reliability Societies, July/August 2015.

13. Keshav Sood, Shui Yu, and Yong Xiang, "Software-Defined Wireless Networking Opportunities and Challenges Internet-of-Things: A Review", IEEE Internet of Things Journal, Vol. 3, No. 4, August 2016.

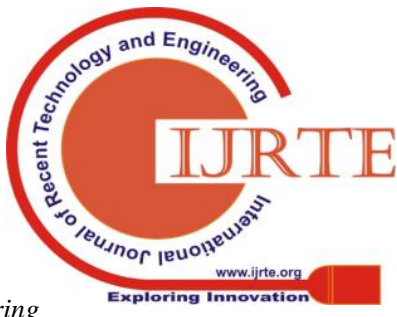
\& Sciences Publication 
14. Michele Nitti, Virginia Pilloni, Giuseppe Colistra, and Luigi Atzori, "The Virtual Object as a Major Element of the Internet of Things," IEEE Communications Surveys \& Tutorials, Vol. 18, No. 2, Second Quarter 2016.

15. Mohammad Abdur Razzaque, Marija Milojevic-Jevric, Andrei Palade, and Siobhán Clarke, "Middleware for Internet of Things: A Survey", IEEE Internet of Things Journal, Vol. 3, No. 1, February 2016.

16. Maria Rita Palattella, Mischa Dohler, and Alfredo Grieco, "Internet of Things in the 5G Era: Enablers, Architecture, and Business Models", IEEE Journal of Selected Areas in Communications, Vol. 34, No. 3, March 2016.

17. Mohamed Essaid Khanouche, Yacine Amirat, Abdelghani Chibani, Moussa Kerkar, and Ali Yachir, "Energy-Centered and QoS-Aware Services Selection for Internet of Things", IEEE Transactions on Automation Science and Engineering, Vol. 13, No. 3, July 2016.

18. Oladayo Bello and Sherali Zeadally, "Intelligent Device-to-Device Communication in the Internet of Things", IEEE Systems Journal, Vol. 10, No. 3, September 2016.

19. Phillip A. Laplante and Nancy Laplante, "The Internet of Things in Healthcare: Potential Applications and Challenges", IT Pro, IEEE Computer Society, May/June 2016.

20. Pawani Porambage, Mika Ylianttila, Corinna Schmitt, Pardeep Kumar, Andrei Gurtov, and Athanasios V. Vasilakos, "The Quest for Privacy in the Internet of Things", IEEE Cloud Computing, March/April 2016.

21. Phillip A. Laplante, Jefrey Voas, and Nancy Laplante, "Standards for the Internet of Things: A Case Study in Disaster Response", IEEE Computer Society, May 2016.

22. Sara Amendola, Rossella Lodato, Sabina Manzari, Cecilia Occhiuzzi, and Gaetano Marrocco, "RFID Technology for IoT-Based Personal Healthcare in Smart Spaces", IEEE Internet of Things Journal, Vol. 1, No. 2, April 2014

23. Yi Xu and Abdelsalam Helal, "Scalable Cloud-Sensor Architecture for the Internet of Things", IEEE Internet of Things Journal, Vol. 3, No. 3, June 2016.

24. Yunchuan Sun, Houbing Song, Antonio J. Jara, and Rongfang Bie, "Internet of Things and Big Data Analytics for Smart and Connected Communities", Digital Object Identifier 10.1109/Access, March 2016.

25. Yuvraj Agarwal and Anind K. Dey, "Toward Building a Safe, Secure, and Easy-to-Use Internet of Things Infrastructure", IEEE Computer Society, April 2016

26. Zhangbing Zhou, Beibei Yao, Riliang Xing, Lei Shu, and Shengrong Bu, "E-CARP: An Energy-Efficient Routing Protocol for UWSNs in the Internet of Underwater Things", IEEE Sensors Journal, Vol. 16, No. 11, June 2016.

27. S.P. Raja, T. Dhiliphan Rajkumar and Vivek Pandiya Raj, Internet of Things: Challenges, Issues and Applications, Journal of Circuits, Systems and Computers, Vol. 27, No. 12, 2018.

28. S.P. Raja, T. Sampradeepraj, Internet of Things: a Research oriented Introductory, International Journal of $\mathrm{Ad} \mathrm{Hoc} \mathrm{and} \mathrm{Ubiquitous}$ Computing, Vol. 29, No. 1/2, 2018.

29. Rajesh, M., and J. M. Gnanasekar. "Path Observation Based Physical Routing Protocol for Wireless Ad Hoc Networks." Wireless Personal Communications 97.1 (2017): 1267-1289.

30. Rajesh, M., and J. M. Gnanasekar. "Sector Routing Protocol (SRP) in Ad-hoc Networks." Control Network and Complex Systems 5.7 (2015): 1-4.

31. Rajesh, M. "A Review on Excellence Analysis of Relationship Spur Advance in Wireless Ad Hoc Networks." International Journal of Pure and Applied Mathematics 118.9 (2018): 407-412.

32. Rajesh, M., et al. "SENSITIVE DATA SECURITY IN CLOUD COMPUTING AID OF DIFFERENT ENCRYPTION TECHNIQUES." Journal of Advanced Research in Dynamical and Control Systems 18.

33. Rajesh, M. "A signature based information security system for vitality proficient information accumulation in wireless sensor systems." International Journal of Pure and Applied Mathematics 118.9 (2018): 367-387.

34. Rajesh, M., K. Balasubramaniaswamy, and S. Aravindh. "MEBCK from Web using NLP Techniques." Computer Engineering and Intelligent Systems 6.8: 24-26. 